\title{
MG132 proteasome inhibitor upregulates the expression of connexin 43 in rats with adriamycin-induced heart failure
}

\author{
GUIYING CHEN**, JIYI ZHAO*, CHUNYAN LIU, YINA ZHANG, YANPING HUO and LIJUN ZHOU \\ Department of Cardiology, The First Affiliated Hospital of Harbin Medical University, \\ Harbin, Heilongjiang 150001, P.R. China
}

Received October 6, 2014; Accepted June 23, 2015

DOI: $10.3892 / \mathrm{mmr} .2015 .4337$

\begin{abstract}
The connexin 43 (Cx43) gap junction protein is important in the synchronization of contraction of cardiac myocytes. Abnormal expression of $\mathrm{Cx} 43$ contributes to ventricular arrhythmia, which is the major cause of sudden death in heart failure (HF). Cx43 is known to interact with zonula occludens ( $\mathrm{ZO})-1$, and the proteasome is involved in the regulation of $\mathrm{Cx} 43$ degradation. Although $\mathrm{Cx} 43$ is downregulated in heart failure, the underlying mechanisms remain to be elucidated. The present study aimed to investigate the effect of the MG132 proteasome inhibitor on the expression levels of $\mathrm{Cx} 43, \mathrm{ZO}-1,20 \mathrm{~S}$ proteasome and ubiquitin in a rat model of $\mathrm{HF}$, induced by adriamycin. MG132 reduced adriamycin-induced injury in the failing heart. In addition, MG132 inhibited the expression of $20 \mathrm{~S}$ proteasome and ubiquitin, accompanied by an upregulation in the expression of $\mathrm{Cx} 43$ and ZO-1. These findings suggested that inhibition of the ubiquitin-proteasome system upregulated the expression of $\mathrm{Cx} 43$. Therefore, the proteasome inhibitor may be used to prevent degradation of $\mathrm{Cx} 43$ in HF, and thus may prevent $\mathrm{Cx} 43$-mediated arrhythmia in $\mathrm{HF}$.
\end{abstract}

\section{Introduction}

Heart failure (HF) is a clinical syndrome, which can result from any heart diseases that lead to inability of the ventricle to pump sufficient blood to meet the body's metabolic demands. HF is associated with a high incidence of sudden death, primarily from ventricular arrhythmia (1). Although the mechanisms underlying ventricular arrhythmia in HF remain

Correspondence to: Dr Lijun Zhou, Department of Cardiology, The First Affiliated Hospital of Harbin Medical University, 23 Youzheng Street, Nangang, Harbin, Heilongjiang 150001, P.R. China

E-mail: lijunzhou12345@outlook.com

${ }^{*}$ Contributed equally

Key words: connexin 43, zonula occludens-1, ubiquitin, proteasome, heart failure to be fully elucidated, it is known that gap junctions, which are unique intercellular channels that directly connect adjacent cardiac myocytes by providing electrical and chemical communication, contribute to various cardiac arrhythmia (2). Gap junctions are composed of connexins, and the number, distribution, and composition of the connexins are important in regulation of the conductive properties of cardiac myocytes (3).

Connexin 43 (Cx43) is the major connexin protein in the ventricle, which is predominantly located in the intercalated disk region between cardiac myocytes in the adult heart. Several lines of evidence have demonstrated that the expression and localization of $\mathrm{Cx} 43$ are altered in the diseased myocardium (2). Downregulation of $\mathrm{Cx} 43$ has been observed in an animal model of HF and in the failing human heart (4-6). In hypertrophic cardiomyopathy and myocardial ischemia, the expression of Cx43 is downregulated (4), and shifts between an end-to-end location and a lateral location $(7,8)$. Furthermore, the heterogeneous loss of $\mathrm{Cx} 43$ in non-ischemic dilated cardiomyopathy is associated with ventricular tachycardia (9).

Zonula occludens (ZO)-1 is a scaffolding protein, which provides a structural basis for assembling protein complexes and maintaining the polarity of epithelial cells (10). In cardiac myocytes, ZO-1 is located in the intercalated disc and the lateral membrane (11-13). Several studies have reported that ZO-1 is directly associated with $\mathrm{Cx} 43$ (14), and regulates the number, distribution and function of $\mathrm{Cx} 43$ (15-17). Furthermore, it has been reported that the expression of ZO-1 is reduced in the failing human heart, accompanied by a decrease in the expression of Cx43 $(18,19)$, suggesting that ZO-1 is important in the downregulation of $\mathrm{Cx} 43$ in $\mathrm{HF}$.

It has been reported that the ubiquitin-proteasome system is involved in the degradation of Cx43 (20). Several studies have revealed that the internalization of $\mathrm{Cx} 43$ is dependent on the proteasomal activities (20-22). Furthermore, ubiquitination of $\mathrm{Cx} 43$ at the cytoplasmic membrane promotes the internalization and subsequent degradation of $\mathrm{Cx} 43$ in a proteasome-dependent manner $(21,23)$. In addition, proteasomes can regulate the interaction between $\mathrm{Cx} 43$ and $\mathrm{ZO}-1$, thus leading to an increase in Cx43 degradation (24). The proteasome inhibitor, MG132, has been observed to inhibit the internalization and degradation of $\mathrm{Cx} 43$ (24,25). However, whether proteasome inhibition can alter the expression of Cx43 in failing hearts remains to be elucidated. 
The present study aimed to investigate the effect of the proteasome inhibitor MG132 on the expression levels of Cx43, ZO-1, 20S proteasome and ubiquitin in a rat model of $\mathrm{HF}$, induced by adriamycin. The purpose of this investigation was to examine the role of inhibition of the ubiquitin-proteasome system by MG132 in the expression of Cx43 in the HF rat model. The results of this investigation may determine whether inhibition of the ubiquitin-proteasome system offers a potential therapeutic strategy for improving the expression of $\mathrm{Cx} 43$ and preventing $\mathrm{Cx} 43$-mediated ventricular arrhythmia in HF.

\section{Materials and methods}

Animals. All experimental procedures were approved by the ethics committee of Harbin Medical University (Harbin, China). Animals were obtained from the Animal Care Center of Harbin Medical University. A total of 70 male Wistar rats (weighing 180-300 g) were housed separately in an atmosphere containing $60 \%$ humidity, with a $12 \mathrm{~h} \mathrm{light/dark}$ cycle at $23 \pm 2^{\circ} \mathrm{C}$. The rats were fed standard chow and water ad libitum.

Adriamycin-induced heart failure model. HF was induced via intraperitoneal injections of adriamycin $(2.5 \mathrm{mg} / \mathrm{kg}$; Hisun Pharmacuetical Co. Ltd., Taizhou, China) to a cumulative dose of $15 \mathrm{mg} / \mathrm{kg}$ in six injections. A total of 70 rats received the first three injections of adriamycin every 3 days, with one injection per day followed by three injections every week. Prior to each drug administration, the body weight of the animal was measured for recalculating dosage. Following the last adriamycin injection, 14 rats had died, with 56 rats remaining alive. The 56 rats were randomly assigned into two groups: Heart failure (HR) group $(n=28)$ and MG132 group $(n=28)$. The rats in the MG132 group received an intraperitoneal injection of MG132 (0.1 mg/kg; Sigma-Aldrich, St. Louis, MO, USA) for 14 days. The rats in the HR group were injected at the same time with the same volume of saline. In addition, normal rats $(n=28)$ without adriamycin injections were used as controls.

Echocardiographic examination. Echocardiographic examination was performed two weeks following the injections of MG132 or saline, using a VIVID 7 dimension system (General Electric, Milwaukee, WI, USA). The rats ( $\mathrm{n}=14$ per group) were anesthetized by intraperitoneal injection of $10 \%$ chloral hydrate (300 mg/kg; Sigma-Aldrich, St. Louis, MO, USA) and the animals were placed in a supine position. The $10 \mathrm{~S}$ transducer $(8 \mathrm{MHz})$ was placed directly on the shaved chest wall and the image depth was adjusted $2-4 \mathrm{~cm}$. The M-mode echocardiogram was used to measure the left ventricle end-systolic diameter (LVESD) and left ventricle end-diastolic diameter (LVEDD) of three consecutive heart cycles, and the average was used for calculation of the ejection fraction (EF) and fractional shortening (FS), as previously reported (26).

Sample collection. Following the final injections of MG132 and saline, the rats ( $\mathrm{n}=14$ per group) were sacrificed by intraperitoneal injection of $10 \%$ chloral hydrate $(300 \mathrm{mg} / \mathrm{kg})$, and the hearts were immediately removed and rinsed in saline. Subsequently, two tissue blocks were obtained from the free wall of the left ventricle, along the long axis of the heart, one of which was fixed in 4\% paraformaldehyde (Sigma-Aldrich) and embedded in paraffin (Sigma-Aldrich). Transverse sections ( $5 \mu \mathrm{m}$ thick) were obtained from paraffin-embedded tissue blocks for hematoxylin and eosin (H\&E) staining (Shanghai Ruiqi Bio-Technology Co., Ltd., Shanghai, China) and immunohistochemistry. The other tissue block was fixed in 3\% glutaraldehyde (Sigma-Aldrich) and used for electron microscopy. The remaining left ventricle tissues were stored at $-80^{\circ} \mathrm{C}$ and used for western blot analysis.

$H \& E$ staining and immunohistochemical analysis. The paraffin-embedded tissue sections were washed in xylene (Sigma-Aldrich) to remove the paraffin, and rehydrated with serial dilutions of alcohol, followed by a wash in phosphate-buffered saline solution (GE Healthcare Life Sciences, Shanghai, China). For the H\&E staining, the sections were stained with H\&E (Beyotime Institute of Biotechnology, Haimen, China). For immunohistochemistry, rthe sections were treated with $3 \% \mathrm{H}_{2} \mathrm{O}_{2}$ (Sigma-Aldrich) at room temperature for $10 \mathrm{~min}$ to block endogenous peroxidase activity. The sections were then incubated in 5\% normal goat serum to block nonspecific protein binding sites. The sections were then incubated with primary antibodies against $\mathrm{Cx} 43$ (polyclonal rabbit anti-rat Cx43; 1:50; cat. no. SC-9059; Santa Cruz Biotechnology, Inc., Santa Cruz, CA, USA), ZO-1 (polyclonal goat anti-rat ZO-1; 1:50; cat. no. SC-8146; Santa Cruz Biotechnology, Inc.), 20 S Proteasome $\alpha 3$ (polyclonal rabbit anti-rat 20S Proteasome a3; 1:5; cat. no. SC-67340; Santa Cruz Biotechnology, Inc.) and ubiquitin (polyclonal rabbit anti-rat ubiquitin; 1:50; cat. no. SC-9133; Santa Cruz Biotechnology, Santa Cruz Biotechnology, Inc.) overnight at $4^{\circ} \mathrm{C}$. Subsequently, the primary antibody was washed off, and the sections were incubated with goat anti-rabbit (HaiGene Biotechnology, Harbin, China) or donkey anti-goat (Santa Cruz Biotechnology, Inc.) biotin-conjugated secondary antibodies $(1: 1,000)$ for $20 \mathrm{~min}$ at $37^{\circ} \mathrm{C}$. The sections were then incubated with streptavidin horseradish peroxidase for $20 \mathrm{~min}$ at $37^{\circ} \mathrm{C}$. The 3, 3-diaminobenzidine (Sigma-Aldrich) substrate was then applied to the sections, which were then counterstained with hematoxylin. Sections in which the primary antibodies were omitted were used as negative controls. The immunostained sections were examined under a light microscope (Olympus IX-51; Olympus Corporation, Tokyo, Japan).

Electron microscopy. The tissue blocks of the left ventricle were fixed with $3 \%$ glutaraldehyde. They were dehydrated in a graded series of ethanol (50, 70, 90 and $100 \%$ for $10 \mathrm{~min}$ each), and then embedded in a 1:1 mixture of Epon812 (Sigma-Aldrich) and acetone (Sigma-Aldrich) for $1 \mathrm{~h}$ at room temperature, followed by embedment in Epon 812 for $2 \mathrm{~h}$. Ultrathin sections (50-100 nm) were cut, stained with lead citrate (Polysciences Inc., Warrington, PA, USA) and uranyl acetate (Sigma-Aldrich). The sections were then viewed and images were captured using an electron microscope (JEM-1200EX; JEOL, Ltd., Tokyo, Japan).

Western blot analysis. The ventricles were homogenized on ice in lysis buffer (Nanjing Sunshine Biotechology Co., Ltd., Nanjing, China). The proteins were resolved by $15 \%$ SDS-PAGE, and transferred onto polyvinylidene 
fluoride membranes (Sigma-Aldrich) by electroblotting. The membranes were incubated with primary antibodies against Cx43 (polyclonal rabbit anti-rat Cx43, 1:500; Santa Cruz Biotechnology, Inc.), ZO-1 (polyclonal goat anti-rat ZO-1; 1:400; Santa Cruz Biotechnology, Inc.), 20S Proteasome $\alpha 3$ (polyclonal rabbit anti-rat 20S Proteasome $\alpha 3$; 1:40; Santa Cruz Biotechnology, Inc) and ubiquitin (polyclonal rabbit anti-rat ubiquitin; 1:400; Santa Cruz Biotechnology, Inc.) at room temperature for $30 \mathrm{~min}$. $\beta$-actin was used as a loading control. The membranes were then incubated with horseradish peroxidase-linked goat anti-rabbit (haiGene Biotechnology) or donkey anti-goat (Santa Cruz Biotechnology, Inc,.) secondary antibodies at room temperature for $20-30 \mathrm{~min}$. The bands were visualized using a chemiluminescence detection system (Invitrogen Life Technologies, Carlsbad, CA, USA) and analyzed using BandScan5.0 (Glyko, Inc., Novato, CA, USA). The expression levels of Cx43, ZO-1, 20S Proteasome and ubiquitin were normalized to that of $\beta$-actin.

Statistical analysis. Analyses were performed using SPSS 17.0 (SPSS Inc., Chicago, IL, USA). All values are presented as the mean \pm standard deviation. One-way analysis of variance was used to compare the differences among groups. $\mathrm{P}<0.05$ were considered to indicate a statistically significant difference.

\section{Results}

MG132 treatment reduces adriamycin-induced cardiotoxicity. The EF and FS were significantly lower in the HF and MG132 groups, compared with the control group $(\mathrm{P}<0.05$; Table I). Although the EF and FS were marginally higher in the MG132 group, compared with the HF group, no significant difference was observed between the two groups ( $>>0.05$; Table I). These data indicated the presence of myocardial damage and suggested that the cardiac contractile function was reduced in adriamycin-induced HF.

Histological examination of the left ventricle in the control group demonstrated that the myocardial fibers were orderly arranged and exhibited homogenously-stained cytoplasm. No damaged fibers were observed, and no edema or exudation were observed. By contrast, vacuolar degeneration and lysis of cardiac myocytes, rupture of myocardial fibers, interstitial edema and infiltration of inflammatory cells were observed in the HF group. Adriamycin-induced damage was reduced in the MG132 group (Fig. 1).

The electron microscopic findings of cardiac myocytes in the control, HF, and MG132 groups were compared (Fig. 2). In the control group, the fine band structure of the sarcomere was clearly identified. The mitochondria were intact and exhibited clear and dense cristae. Desmosomes, gap junctions and intermediate junctions were clearly visible (Fig. 2A and B). By contrast, in the HF group, lysis of the myocardial fibers and disappearance of the band structure of the sarcomere were observed. The remaining myofibrils were disorganized, and the lysed myofibrils were filled with a numerous cell organelles. An increased number of lysosomes were present between the mitochondria, and swollen mitochondria with loss of cristae were found. High electron-dense particles were observed in the nucleus, with disappearance of organelles at the paranuclear region. The gap junctions were disorganized
Table I. EF and FS in the control, HF and MG132 groups.

\begin{tabular}{lccc}
\hline Factor & Control $(\%)$ & HF $(\%)$ & MG132 $(\%)$ \\
\hline EF & $82.15 \pm 3.57$ & $69.65 \pm 4.65^{\mathrm{a}}$ & $67.21 \pm 7.13^{\mathrm{a}}$ \\
FS & $45.25 \pm 3.12$ & $33.78 \pm 3.35^{\mathrm{a}}$ & $31.85 \pm 4.15^{\mathrm{a}}$
\end{tabular}

${ }^{\mathrm{a}} \mathrm{P}<0.05$, vs. control. Values are presented as the mean \pm standard deviation. HF, heart failure; EF, ejection fraction; FS, fractional shortening.

and formed a tube-like structure. In the MG132 group, the band structure of the sarcomere was clearly visible. The sarcoplasmic reticulum was marginally enlarged. The mitochondria were intact, however, the number of mitochondria containing flocculent densities inside was decreased. Desmosomes, gap junctions and intermediate junctions were intact.

MG132 increases the expression of Cx43 and ZO-1 in $H F$ rats. The present study further examined the effect of MG132 on the expression levels of Cx43 and ZO-1 in HF rats, using immunohistochemistry and western blot analysis. Immunohistochemical investigations demonstrated that the expression of $\mathrm{Cx} 43$ was lower in the HF group, compared with the control group. MG132 treatment increased the expression of $\mathrm{Cx} 43$ in the HF rats (Fig. 3A-C). Consistent with the immunohistochemical results, the results of the western blot analysis demonstrated that the expression of $\mathrm{Cx} 43$ was significantly decreased in the HF group, compared with the control group, and MG132 treatment increased the expression of Cx43 in the HF rats (Fig. 3D).

The immunohistochemical results revealed that $\mathrm{ZO}-1$ was widely expressed in each group (Fig. 4). The intensity of ZO-1 immunostaining was weaker in the HF group, compared with the control group, and was more marked in the MG132 group, compared with the HF group (Fig. 4A-C). Consistent with these findings, western blot analysis revealed that the expression of ZO-1 was significantly decreased in the HF group, compared with the control group, and significantly increased in the MG132 group, compared with the HF group (Fig. 4D).

MG132 donwregulates the expression levels of 20 S proteasome and ubiquitin in HF rats. The present study then investigated the effect of MG132 on the expression levels of 20 S proteasome and ubiquitin in the HF rats, using immunohistochemistry and western blot analysis. The immunohistochemical analysis demonstrated that $20 \mathrm{~S}$ proteasome was widely expressed in each group (Fig. 5A-C). The expression of proteasome-positive cells was higher in the HF group, compared with the control group, and MG132 treatment downregulated the expression of proteasome in the HF rats (Fig. 5A-C). Consistent with the immunohistochemistry, western blot analysis revealed that the expression of proteasome was significantly increased in the HF group, compared with the control group, and that MG132 treatment decreased the expression of proteasome in the $\mathrm{HF}$ rats (Fig. 5D).

The immunohistochemical results revealed that ubiquitin was predominantly expressed in the nuclei of the cells in each 
A

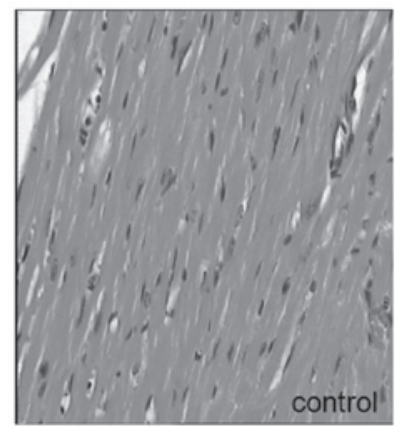

B

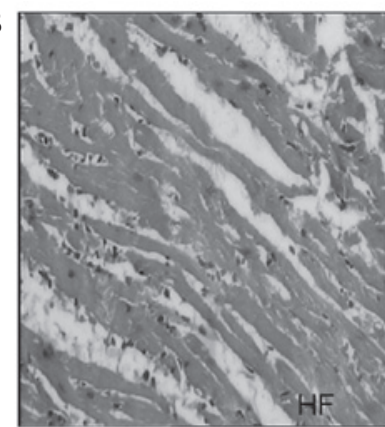

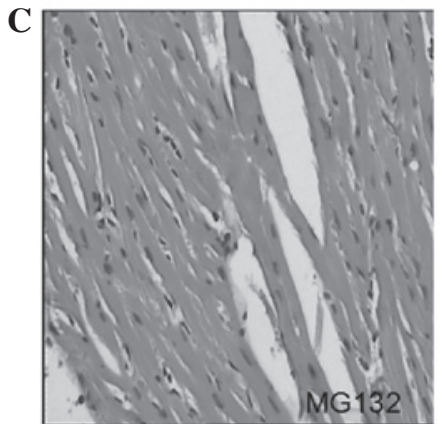

Figure 1. Hematoxlin and eosin staining of the left ventricle in the (A) control, (B) HF and (C) MG132 groups. Magnification x200. (A) The myocardial fibers in the control group were arranged and exhibited homogeneously stained cytoplasm. (B) The ventricle in the HF group exhibited vacuolar degeneration and lysis of cardiac myocytes, rupture of myocardial fibers, interstitial edema, and infiltration of inflammatory cells. (C) The ventricle in the MG132 group exhbited mild damage. HF, heart failure; Cx43, connexin 43.
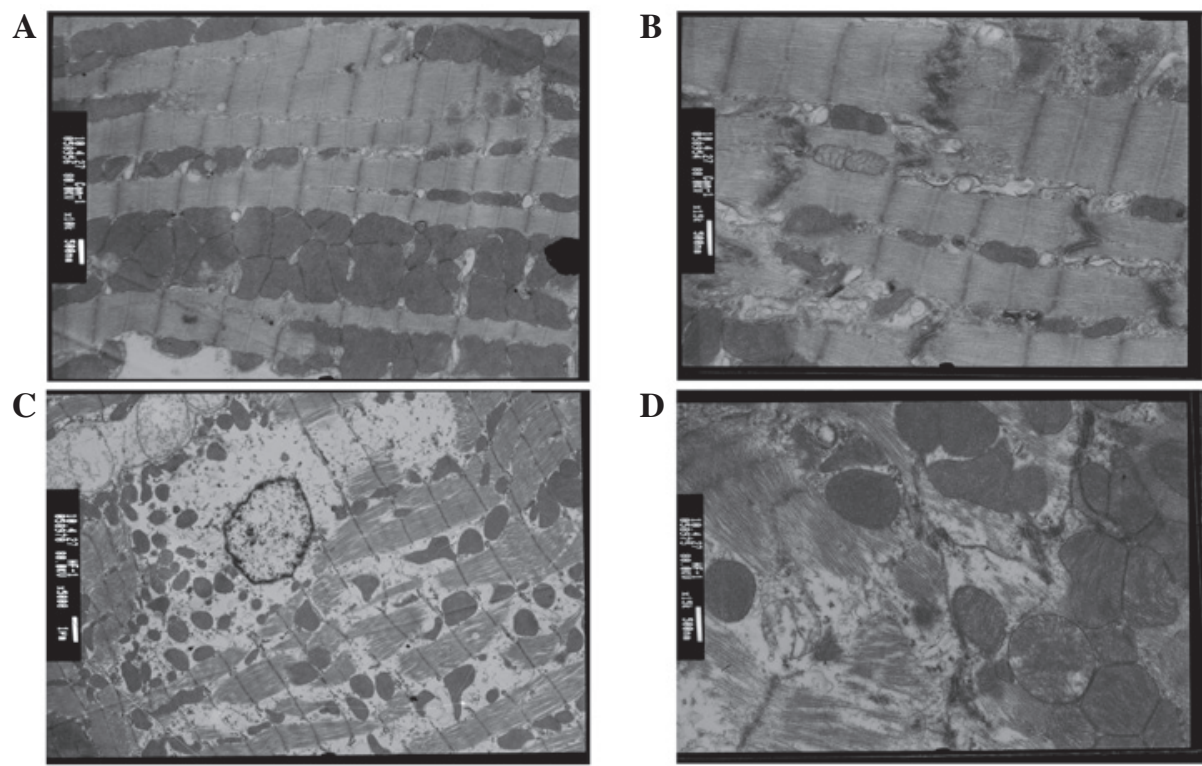

D
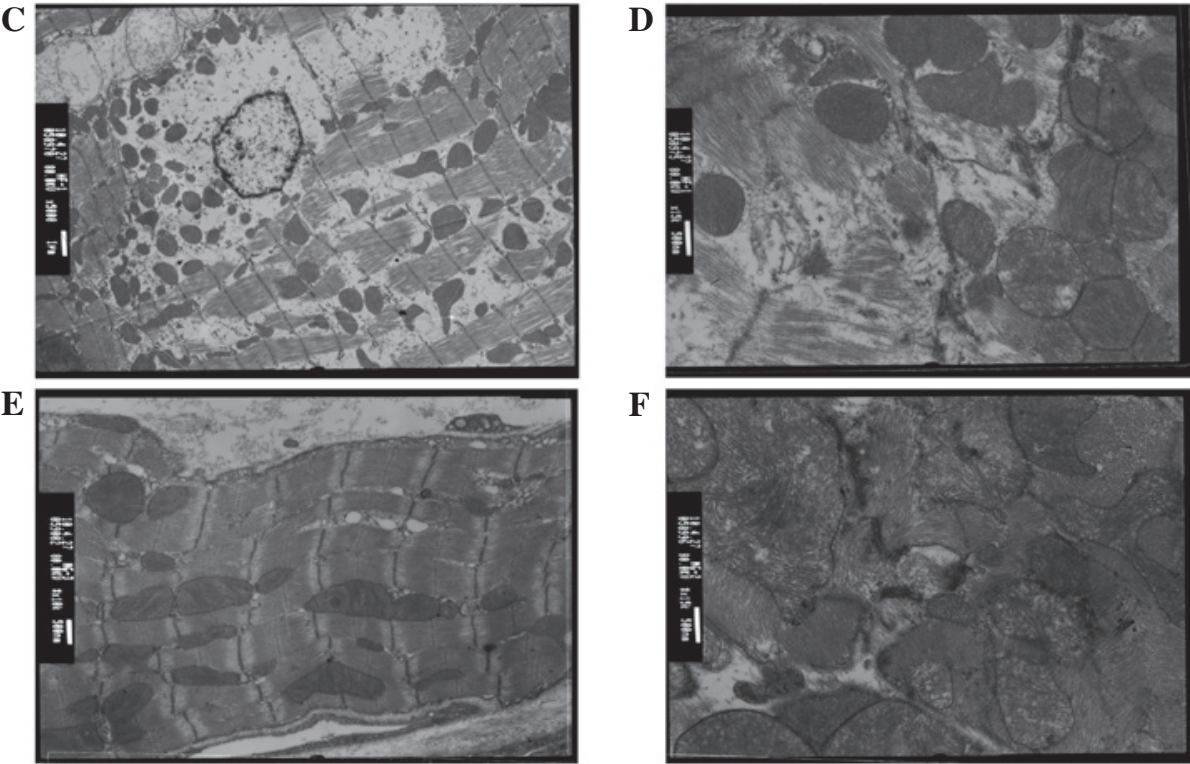

Figure 2. Electron microscopy images of cardiac myocytes in the (A and B) control, (C and D) HF, and (E and F) MG132 groups. (A an B) The fine band structure of the sarcomere is clearly visible with intact mitochondria, desmosomes, gap junctions, and intermediate junctions. (C and D) Lysis of myocardial fibers, disappearance of sarcomere band structure, and disorganized myofibrils are visible with a high number of lysosomes, swollen mitochondria and high electron-dense particles. In addition, the gap junctions are disorganized. (E and F) The band structure of the sarcomere is clearly visible with partially enlarged sarcoplasmic reticulum and intact mitochondria, desmosomes, gap junctions, and intermediate junctions. Magnification: A,C and E, x10,000; B, D, and $\mathrm{F}, \mathrm{x} 15,000$. HF, heart failure; Cx43, connexin 43 .

group (Fig. 6A-C). The intensity of ubiquitin immunostaining was more marked in the HF groupn compared with the control group, and was weaker in the MG132 group, compared with the HF group (Fig. 6A-C). Consistent with this, western blot analysis revealed that the expression of ubiquitin was significantly increased in the HF group, compared with the control group, and was decreased in the MG132 group, compared with the HF group (Fig. 6D).

\section{Discussion}

Gap junctions have been well known to contribute to cardiac arrhythmia (2), which is a predominant cause of sudden death in HF. Previous animal and clinical studies have found downregulation of $\mathrm{Cx} 43$ in failing hearts (4-6). Although several lines of evidence have demonstrated that proteasomal activities are required for the degradation of $\mathrm{Cx} 43$, whether 

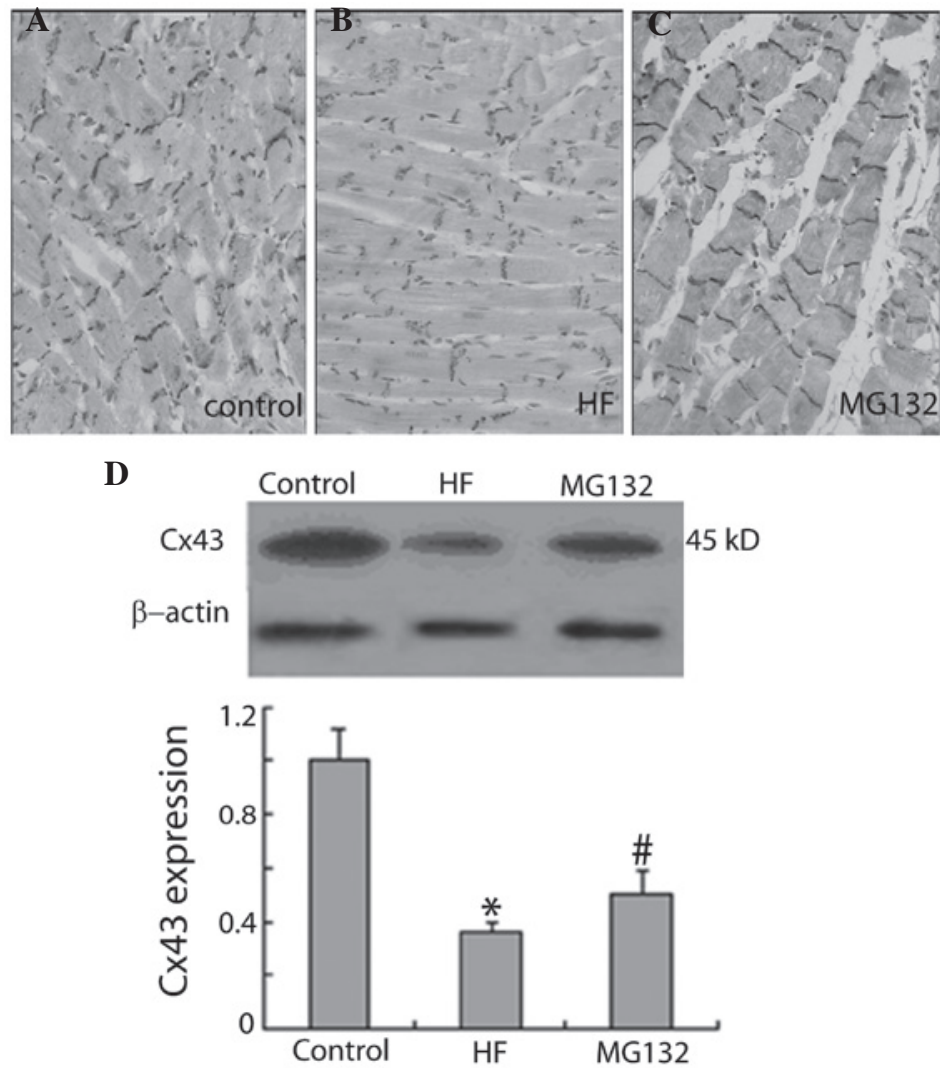

Figure 3. MG132 increases the expression of Cx43 in HF rats. Representative immunohistochemical staining of Cx43 in the (A) control (B) HF and (C) MG132 groups, demonstrating that the expression levels of $\mathrm{Cx} 43$ are decreased in the HF group, and increased in the MG132 group. (D) Western blot analysis of the expression of $\mathrm{Cx} 43$ in the control, HF and MG132 groups. $\beta$ actin was used as a loading control. The expression level of Cx43 was normalized to that of $\beta$ actin. The ratio of the $\mathrm{Cx} 43$ to in the control group was set as 1 . Values are presented as the mean \pm standard deviation $(\mathrm{n}=3)$. ${ }^{*} \mathrm{P}<0.05$, vs. control; ${ }^{*} \mathrm{P}<0.05$, vs. HF. HF, heart failure; Cx43, connexin 43.
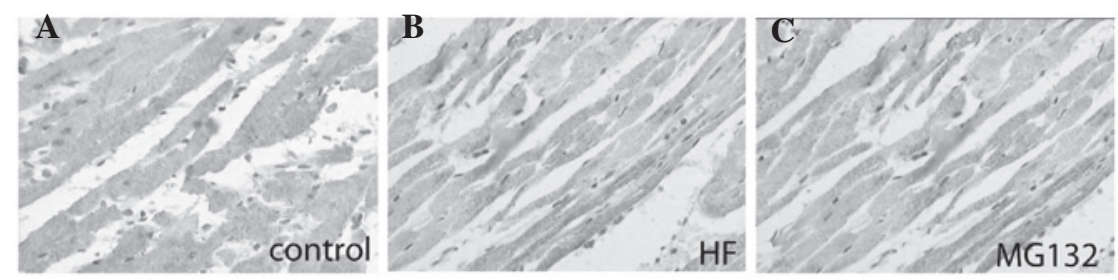

D
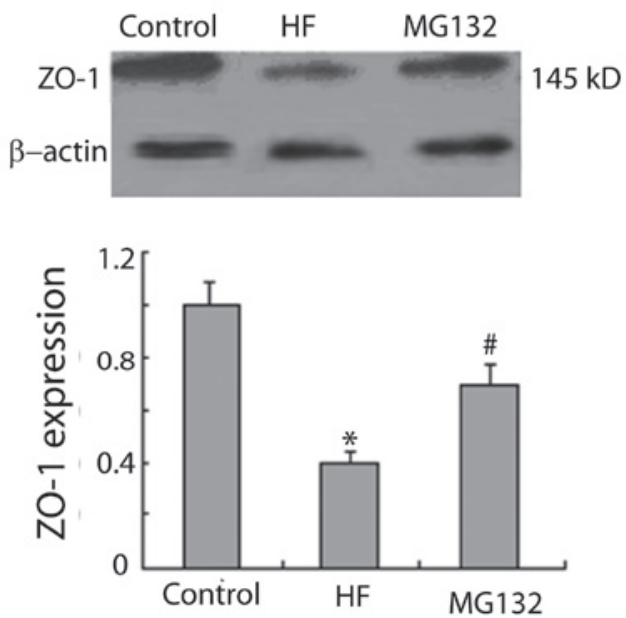

Figure 4. MG132 increases the expression of ZO-1 in HF rats. Representative immunohistochemical staining of ZO-1 in the (A) control (B) HF and (C) MG132 groups, demonstrating that the expression levels of ZO-1 were decreased in the HF group, and increased in the MG132 group. (D) Western blot analysis of the expression of ZO-1 in the control, HF and MG132 groups. $\beta$ actin was used as a loading control. The expression level of ZO-1 was normalized to that of $\beta$-actin. The ratio of the ZO-1 to in the control group was set as 1 . Values are presented as the mean \pm standard deviation $(\mathrm{n}=3)$. ${ }^{*} \mathrm{P}<0.05$, vs. control; ${ }^{\#} \mathrm{P}<0.05$, vs. HF. HF, heart failure; ZO-1, zonula occludens. 

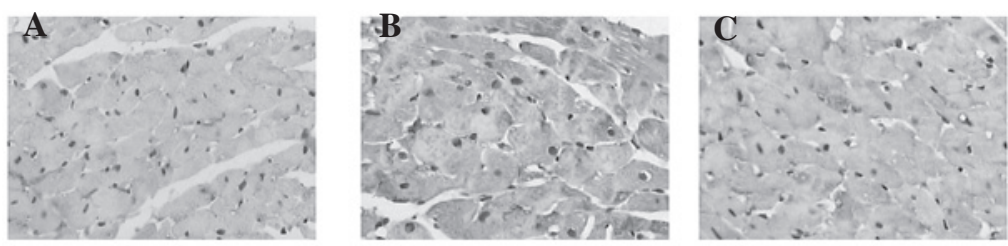

D
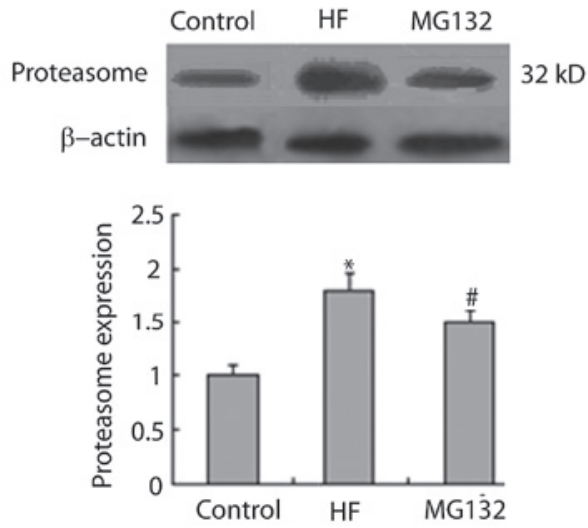

Figure 5. MG132 decreases the expression of 20S proteasome in HF rats. Representative immunohistochemical staining of 20S proteasome in the (A) control (B) HF and (C) MG132 groups, demonstrating that the expression levles of the 20S proteasome were increased in the HF group, and decreased in the MG132 group. (D) Western blot analysis of the expression of $20 \mathrm{~S}$ proteasome in the control, HF and MG132 groups. $\beta$ actin was used as a loading control. The expression level of 20S proteasome was normalized to that of $\beta$ actin. The ratio of the 20S proteasome to in the control group was set as 1 . Values are presented as the mean \pm standard deviation $(\mathrm{n}=3)$. ${ }^{*} \mathrm{P}<0.05$, vs. control; ${ }^{\mathrm{P}} \mathrm{P}<0.05$, vs. HF. $\mathrm{HF}$, heart failure.

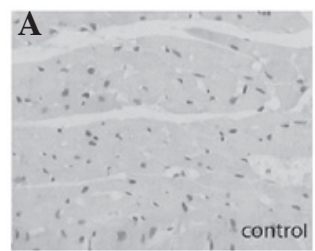

D
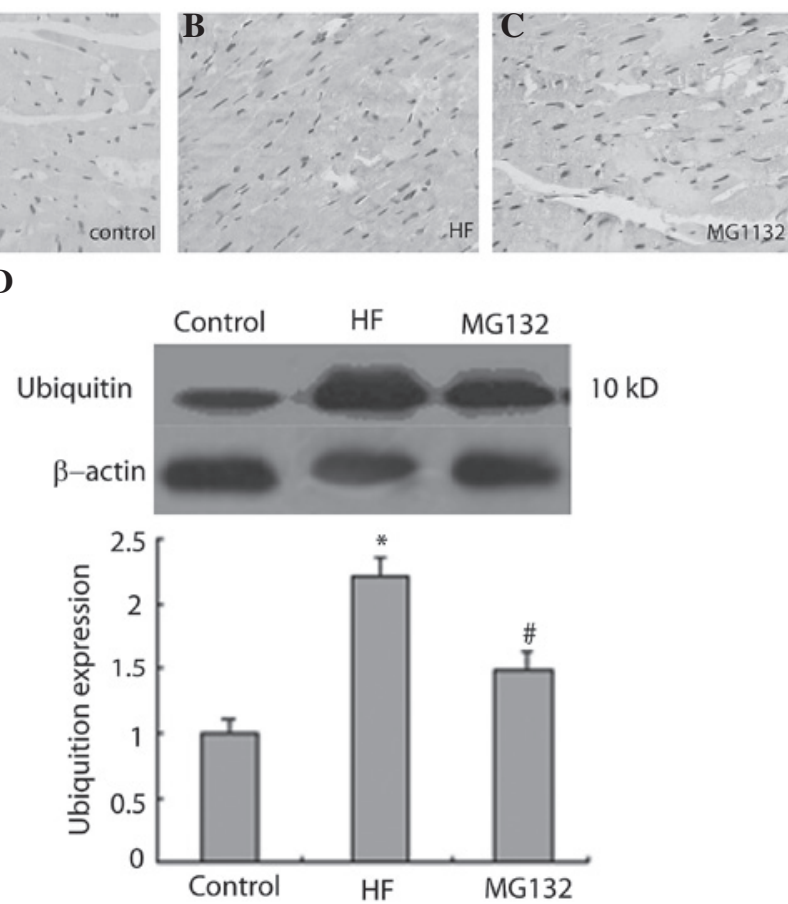

Figure 6. MG132 decreases the expression of ubiquitin in HF rats. Representative immunohistochemical staining of ubiquitin in the (A) control (B) HF and (C) MG132 groups, demonstrating that the expression levels of ubiquitin were increased in the HF group, and decreased in the MG132 group. (D) Western blot analysis of the expression of ubiquitin in the control, HF, and MG132 groups. $\beta$ actin was used as a loading control. The expression level of ubiquitin was normalized to that of $\beta$ actin. The ratio of the ubiquitin to in the control group was set as 1 . Values are presented as the mean \pm standard deviation (n=3). ${ }^{*} \mathrm{P}<0.05$, vs. control; ${ }^{\mathrm{P}}<0.05$, vs. HF. HF, heart failure.

proteasome activities contribute to the downregulation of $\mathrm{Cx} 43$ in HF remain to be elucidated. In the present study, the role of proteasome in regulation of the expression of $\mathrm{Cx} 43$ in rats with $\mathrm{HF}$, induced by adriamycin, was investigated using the MG132 proteasome inhibitor. The results revealed that MG132 inhibited the expression of 20S proteasome and ubiquitin, and reduced adriamycin-induced injury in the heart, accompanied by upregulation in the expression of $\mathrm{Cx} 43$ and 
ZO-1. These findings suggested that the inhibition of proteasome upregulates the expression $\mathrm{Cx} 43$ in $\mathrm{HF}$, and thus may prevent Cx43-mediated arrhythmia.

In the present study, a rat model of heart failure was produced by intraperitoneal injections of adriamycin at a cumulative dose of $15 \mathrm{mg} / \mathrm{kg}$. This treatment resulted in a decrease in the cardiac contractile function, as indicated by a reduction in EF and $\mathrm{FS}$, and an increase in myocardial cell damage, as indicated by $H \& E$ staining and electron microscopy, suggesting that HF was successfully induced by adriamycin. This experimental model has been used previously for investigating HF in rats, and the myocardial lesion induced by adriamycin exhibits histological and hemodynamic features similar to those reported in failing human hearts (27-29). In the present study, MG132 treatment reduced myocardial damage in the HF rats, suggesting that proteasome inhibition may be a therapeutic strategy for the treatment of HF.

Several previous studies have demonstrated that the expression of $\mathrm{Cx} 43$ shifts to the lateral membrane in hypertrophic cardiomyopathy and myocardial ischemia $(7,8)$. Furthermore, it has been reported that $\mathrm{Cx} 43$ is downregulated in an animal model of HF and in the failing human heart (4-6). Consistent with these studies, the present study found that the expression of Cx43 was significantly decreased in the HF rats. In addition, it has been reported that the heterogeneous reduction of Cx43 contributes to the development of malignant ventricular tachycardia (9). In the present study, MG132 increased the expression of Cx43 in HF rats, which was demonstrated using immunohistochemistry and western blot analysis. This observation that MG132 increased the expression of Cx43 in the HF rats suggested that proteasome may mediate the heterogeneous reduction of $\mathrm{Cx} 43$, and that inhibition of proteasome may prevent ventricular tachycardia in HF.

The present study further investigated the expression of ZO-1 in HF rats, and found that the expression of ZO-1 was not restricted to the intercalated disc. Consistent with this finding, several studies have reported that the expression of ZO-1 is located in the intercalated disk and the lateral membrane (11-13). Furthermore, it has been reported that the expression of ZO-1 is reduced in the failing human heart, accompanied by a decrease in the expression of Cx43 $(18,19)$. Consistent with these studies, the present study demonstrated that ZO-1 was downregulated in the HF rats, accompanied by a downregulation of $\mathrm{Cx} 43$, suggesting that ZO-1 may contribute to the downregulation of Cx43 in HF. Several studies have reported that ZO-1 can regulate the number, distribution and function of $\mathrm{Cx} 43$ (15-17). MG132 treatment significantly increased the expression levels of ZO-1 and $\mathrm{Cx} 43$ in the HF rats, suggesting that the proteasome-mediated regulation of the interaction between $\mathrm{Cx} 43$ and ZO-1 may contribute to changes in the expression of $\mathrm{Cx} 43$ in HF. In agreement with this hypothesis, it has been reported that proteasome can regulate the expression of $\mathrm{Cx} 43$ via modulating the interaction of $\mathrm{Cx} 43$ with ZO-1 (24).

The ubiquitin-proteasome system is involved in the internalization and degradation of Cx43 (20-22). It has been reported that the expression and activity of proteasome are increased during chronic pressure overload, thus leading to ventricular hypertrophy (30). In the present study, the expression of $20 \mathrm{~S}$ proteasome and ubiquitin was increased in the HF rats, accompanied by an increase in the expression of $\mathrm{Cx} 43$, suggesting that an increase in the activity of ubiquitin-proteasome system may contribute to the downregulation of $\mathrm{Cx} 43$. Furthermore, the MG132 proteasome inhibitor inhibited the expression of $20 \mathrm{~S}$ proteasome and ubiquitin, and upregulated the expression of $\mathrm{Cx} 43$ in the HF rats, further confirming the role of the ubiquitin-proteasome system in regulating the expression of $\mathrm{Cx} 43$ in $\mathrm{HF}$.

In conclusion, the present study demonstrated that the MG132 proteasome inhibitor inhibited the expression of $20 \mathrm{~S}$ proteasome and ubiquitin, and upregulated the expression of Cx43 and ZO-1 in rats with adriamycin-induced HF. These results suggest that inhibition of the ubiquitin-proteasome system may effectively prevent the degradation of $\mathrm{Cx} 43$ in $\mathrm{HF}$, and thus may reduce the occurrence of ventricular arrhythmia.

\section{Acknowledgements}

The present study was supported by the National Natural Science Foundation of China (grant no. 81300134), the Research Fund for the Doctoral Program of Higher Education of China (grant no. 20112307120014), and the Heilongjiang Province Natural Science Foundation of China (grant no. H201443).

\section{References}

1. Packer M: Sudden unexpected death in patients with congestive heart failure: A second frontier. Circulation 72: 681-685, 1985.

2. Jongsma HJ and Wilders R: Gap junctions in cardiovascular disease. Circ Res 86: 1193-1197, 2000.

3. Saffitz JE, Davis LM, Darrow BJ, Kanter HL, Laing JG and Beyer EC: The molecular basis of anisotropy: Role of gap junctions. J Cardiovasc Electrophysiol 6: 498-510, 1995.

4. Dupont E, Matsushita T, Kaba RA, Vozzi C, Coppen SR, Khan N, Kaprielian R, Yacoub MH and Severs NJ: Altered connexin expression in human congestive heart failure. J Mol Cell Cardiol 33: 359-371, 2001

5. Kostin S, Rieger M, Dammer S, Hein S, Richter M, Klovekorn WP, Bauer EP and Schaper J: Gap junction remodeling and altered connexin43 expression in the failing human heart. Mol Cell Biochem 242: 135-144, 2003

6. Wang X and Gerdes AM: Chronic pressure overload cardiac hypertrophy and failure in guinea pigs: III. Intercalated disc remodeling. J Mol Cell Cardiol 31: 333-343, 1999.

7. Sepp R, Severs NJ and Gourdie RG: Altered patterns of cardiac intercellular junction distribution in hypertrophic cardiomyopathy. Heart 76: 412-417, 1996.

8. Severs NJ, Coppen SR, Dupont E, Yeh HI, Ko YS and Matsushita T: Gap junction alterations in human cardiac disease. Cardiovasc Res 62: 368-377, 2004.

9. Kitamura H, Ohnishi Y, Yoshida A, Okajima K, Azumi H, Ishida A, Galeano EJ, Kubo S, Hayashi Y, Itoh H and Yokoyama M: Heterogeneous loss of connexin43 protein in nonischemic dilated cardiomyopathy with ventricular tachycardia. J Cardiovase Electrophysiol 13: 865-870, 2002.

10. Hahn AF, Ainsworth PJ, Naus CC, Mao J and Bolton CF: Clinical and pathological observations in men lacking the gap junction protein connexin 32. Muscle Nerve Suppl 9 (Suppl): S39-S48, 2000.

11. Gutstein DE, Liu FY, Meyers MB, Choo A and Fishman GI: The organization of adherens junctions and desmosomes at the cardiac intercalated disc is independent of gap junctions. J Cell Sci 116: 875-885, 2003.

12. Li J, Patel VV, Kostetskii I, Xiong Y, Chu AF, Jacobson JT, Yu C, Morley GE, Molkentin JD and Radice GL: Cardiac-specific loss of $\mathrm{N}$-cadherin leads to alteration in connexins with conduction slowing and arrhythmogenesis. Circ Res 97: 474-481, 2005.

13. Sanford JL, Edwards JD, Mays TA, Gong B, Merriam AP and Rafael-Fortney JA: Claudin-5 localizes to the lateral membranes of cardiomyocytes and is altered in utrophin/ dystrophin-deficient cardiomyopathic mice. J Mol Cell Cardiol 38: 323-332, 2005. 
14. Toyofuku T, Yabuki M, Otsu K, Kuzuya T, Hori M and Tada M: Direct association of the gap junction protein connexin-43 with ZO-1 in cardiac myocytes. J Biol Chem 273: 12725-12731, 1998

15. Hunter AW, Barker RJ, Zhu C and Gourdie RG: Zonula occludens-1 alters connexin43 gap junction size and organization by influencing channel accretion. Mol Biol Cell 16: 5686-5698, 2005.

16. Hunter AW, Jourdan J and Gourdie RG: Fusion of GFP to the carboxyl terminus of connexin43 increases gap junction size in HeLa cells. Cell Commun Adhes 10: 211-214, 2003.

17. Jin C, Martyn KD, Kurata WE, Warn-Cramer BJ and Lau AF: Connexin43 PDZ2 binding domain mutants create functional gap junctions and exhibit altered phosphorylation. Cell Commun Adhes 11: 67-87, 2004.

18. Bruce AF, Rothery S, Dupont E and Severs NJ: Gap junction remodelling in human heart failure is associated with increased interaction of connexin 43 with ZO-1. Cardiovasc Res 77: 757-765, 2008

19. Laing JG, Saffitz JE, Steinberg TH and Yamada KA: Diminished zonula occludens-1 expression in the failing human heart. Cardiovasc Pathol 16: 159-164, 2007.

20. Laing JG, Tadros PN, Westphale EM and Beyer EC: Degradation of connexin43 gap junctions involves both the proteasome and the lysosome. Exp Cell Res 236: 482-492, 1997.

21. Leithe E and Rivedal E: Epidermal growth factor regulates ubiquitination, internalization and proteasome-dependent degradation of connexin43. J Cell Sci 117: 1211-1220, 2004.
22. Musil LS, Le AC, VanSlyke JK and Roberts LM: Regulation of connexin degradation as a mechanism to increase gap junction assembly and function. J Biol Chem 275: 25207-25215, 2000.

23. Berthoud VM, Tadros PN and Beyer EC: Connexin and gap junction degradation. Methods 20: 180-187, 2000.

24. Girao $\mathrm{H}$ and Pereira P: The proteasome regulates the interaction between Cx43 and ZO-1. J Cell Biochem 102: 719-728, 2007.

25. Leithe $\mathrm{E}$ and Rivedal E: Ubiquitination and down-regulation of gap junction protein connexin-43 in response to 12-O-tetradecanoylphorbol 13-acetate treatment. J Biol Chem 279: 50089-50096, 2004

26. Borer JS, Jason M, Devereux RB, Fisher J, Green MV, Bacharach SL, Pickering T and Laragh JH: Function of the hypertrophied left ventricle at rest and during exercise. Hypertension and aortic stenosis. Am J Med 75: 34-39, 1983.

27. Mettler FP, Young DM and Ward JM: Adriamycin-induced cardiotoxicity (cardiomyopathy and congestive heart failure) in rats. Cancer Res 37: 2705-2713, 1977.

28. Rabelo E, De Angelis K, Bock P, Gatelli Fernandes T, Cervo F, Belló Klein A, Clausell $\mathrm{N}$ and Cláudia Irigoyen M: Baroreflex sensitivity and oxidative stress in adriamycin-induced heart failure. Hypertension 38: 576-580, 2001.

29. Singal PK, Iliskovic N, Li T and Kumar D: Adriamycin cardiomyopathy: Pathophysiology and prevention. Faseb J 11: 931-936, 1997.

30. Depre C, Wang Q, Yan L, Hedhli N, Peter P, Chen L, Hong C, Hittinger L, Ghaleh B, Sadoshima J, et al: Activation of the cardiac proteasome during pressure overload promotes ventricular hypertrophy. Circulation 114: 1821-1828, 2006. 